\title{
Clinical Outcomes of Immune Checkpoint Blocker Therapy for Malignant Melanoma in Korean Patients: Potential Clinical Implications for a Combination Strategy Involving Radiotherapy
}

\author{
Jeongshim Lee, $M D^{1,2}$ \\ Jee Suk Chang, MD ${ }^{1}$ \\ Mi Ryung Roh, MD, $\mathrm{PhD}{ }^{3}$ \\ Minkyu Jung, MD, PhD ${ }^{4}$ \\ Choong-Kun Lee, $\mathrm{MD}^{4}$ \\ Byung Ho Oh, MD, PhD 3 \\ Kee Yang Chung, $\mathrm{MD}, \mathrm{PhD}^{3}$ \\ Woong Sub Koom, MD, PhD' \\ Sang Joon Shin, MD, $\mathrm{PhD}^{4}$
}

\author{
${ }^{1}$ Department of Radiation Oncology, \\ Yonsei University College of Medicine, Seoul, \\ ${ }^{2}$ Department of Radiation Oncology, \\ Inha University Hospital, Incheon, \\ ${ }^{3}$ Department of Dermatology, \\ ${ }^{4}$ Division of Medical Oncology, \\ Department of Internal Medicine, \\ Yonsei University College of Medicine, \\ Seoul, Korea
}

\section{Purpose}

We investigated the clinical efficacy of immune checkpoint blocker (ICB) therapy for metastatic or advanced melanoma in Korean patients. As well, we assessed whether the effects of ICBs can be enhanced by combination therapy with palliative radiotherapy (RT).

\section{Materials and Methods}

We retrospectively reviewed the records of 127 patients with metastatic melanoma who received ICB with or without palliative RT between 2014 and 2018. The melanoma subtypes were classified as follows: chronic sun-damaged (CSD), acral, mucosal, and uveal. The primary endpoint was the objective response rate (ORR).

\section{Results}

The overall ORR was 15\%, with 11 complete and eight partial responses. ORRs for CSD, acral/mucosal, and uveal melanomas were $50 \%, 16.5 \%$, and $0 \%$, respectively $(p=0.009)$. In addition to the subtype, stage at treatment, total tumor burden at treatment, and ICB type were significantly associated with ORR (all $p<0.05$ ). Palliative RT was administered in $44 \%$ of patients during the treatment, and it did not affect ORR. Clinical responders to ICB therapy exhibited significantly higher 1-year progression-free and overall survival rates than nonresponders.

\section{Conclusion}

ORR for ICB monotherapy in Korean patients with melanoma is relatively modest compared with that in Western patients because the non-CSD subtypes are predominant in the Korean population. Our findings regarding combination therapy with ICB provided a rationale for the initiation of our phase II study (NCT04017897).

\section{Key words}

Melanoma, Immunotherapy, Immune checkpoint blocker, Asia 


\section{Introduction}

The clinical effects of immune checkpoint blockers (ICBs), such as programmed death 1 (PD-1)/ programmed death-ligand 1 (PD-L1) inhibitors and cytotoxic T lymphocyte antigen 4 (CTLA-4) inhibitors are most noticeable in patients with metastatic or advanced melanoma because they contribute to an improvement in progression-free survival (PFS) and overall survival (OS) $[1,2]$. However, most studies have analyzed the efficacy of ICBs in Caucasian patients who primarily develop cutaneous melanoma in response to chronic sun exposure [3,4]. Asian populations, including the Korean population, predominantly develop acral or mucosal melanomas not associated with chronic sun damage and exhibit a low somatic mutational burden [4-6]. Consequently, the effectiveness of ICBs in Asian patients with melanoma remains unclear.

In addition, the efficacy of ICB monotherapy is inadequate as the proportion of nonresponders is $60 \%-70 \%$ [7]. Therefore, combination strategies for enhancing the effectiveness of ICB therapy and broadening its indications are being explored, although the optimal combination strategy remains unclear [8]. Conventional treatments such as chemotherapy, targeted therapy, and radiotherapy (RT) have immunomodulatory effects and may exhibit synergistic potential when combined with ICB therapy [8-11]. Among these, RT could be a good option for combination therapy because it not only facilitates effective local control but also exhibits the so-called abscopal effect (response at a distance from the irradiated volume), which primes immunogenic cell death by generating a cytotoxic adaptive immune response [12].

In this study, we aimed to investigate the clinical efficacy of ICB therapy for metastatic or advanced melanoma in Korean patients. Additionally, we assessed whether the effects of ICBs in these patients can be enhanced by combination therapy with palliative RT.

\section{Materials and Methods}

\section{Patient population}

We retrospectively identified consecutive Korean patients with advanced or metastatic malignant melanoma treated with ICBs between 2014 and 2018 at Yonsei Cancer Center, Seoul, Republic of Korea. Data regarding patient demographics, treatments and related parameters, and outcomes were obtained from the medical records. The inclusion criteria were as follows: age $\geq 18$ years; pathologically confirmed malignant melanoma; at least one cycle of treatment with a CTLA-4 inhibitor or a PD-1/ PD-L1 inhibitor; and availability of follow-up images for the assessment of treatment response. The tumors were clinically classified according to the extent of surrounding solar elastosis and the anatomical site as chronic sun-damaged (CSD) melanomas and non-CSD melanomas (acral, mucosal, and uveal). The use of palliative RT during the course of treatment was evaluated for all patients. For advanced or metastatic malignant melanoma, palliative RT was administered for symptomatic metastasis after thorough discussion with a multidisciplinary melanoma team. Combination therapy with ICB and RT was defined as the administration of RT during ICB therapy or within 3 months before and after ICB therapy. The interval period of 3 months was based on five half-lives of the administered ICB; this ensured that $97 \%$ of the drug would be eliminated from the body [13].

\section{Outcome assessments}

The follow-up period was defined as the interval between the first ICB administration and the date of the last visit or death. The primary endpoint was the objective response rate (ORR), including complete response (CR) and partial response (PR), after ICB with or without RT. The radiological response was assessed according to the immune Response Evaluation Criteria in Solid Tumors (iRECIST) [14,15]. ORR was evaluated according to tumor and treatment variables. The secondary endpoints were PFS, defined as the time from ICB administration to disease progression or death, and OS, defined as the time from ICB administration to death from any cause. In addition, ICB- or RT-related adverse events were recorded using the Common Terminology Criteria for Adverse Events, ver. 4.03 .

\section{Statistical analysis}

All statistical analyses were performed using SPSS ver. 20.0 (IBM Corp., Armonk, NY). Chi-square tests, Fisher exact tests, or linear-by-linear associations were used to evaluate ORR according to various tumor and treatment variables in the univariate analysis. Multivariate analysis was performed using a binary logistic regression model employing a backward stepwise method. Survival analyses were performed using the Kaplan-Meier method, and the log-rank test was used for intergroup comparisons. A p-value of $<0.05$ was considered statistically significant.

\section{Ethical statement}

This study was approved by our Institutional Review Board (4-2019-0796). Informed consent was waived. 
Table 1. Baseline characteristics of Korean patients with metastatic melanoma who received immune checkpoint blockers

\begin{tabular}{|c|c|}
\hline Characteristic & Jo. $(\%)(n=127)$ \\
\hline Age at diagnosis, median (range, yr) & $56(18-86)$ \\
\hline Age at ICB administration, median (range, yr) & $60(19-89)$ \\
\hline$<60$ & $60(47.2)$ \\
\hline$\geq 60$ & $67(52.8)$ \\
\hline \multicolumn{2}{|l|}{ Sex } \\
\hline Male & $66(52.0)$ \\
\hline Female & $61(48.0)$ \\
\hline \multicolumn{2}{|l|}{ Subtype } \\
\hline Acral/Mucosal & $103(81.1)$ \\
\hline Uveal & $20(15.7)$ \\
\hline Chronic sun damage & $4(3.1)$ \\
\hline \multicolumn{2}{|l|}{$B R A F$ status } \\
\hline Wild type & $89(70.1)$ \\
\hline V600 mutation & $16(12.6)$ \\
\hline Unknown & $22(17.3)$ \\
\hline \multicolumn{2}{|l|}{ Stage at ICB administration } \\
\hline M1a & $31(24.4)$ \\
\hline M1b & $17(13.4)$ \\
\hline M1c & $66(52.0)$ \\
\hline M1d & $8(6.3)$ \\
\hline Unresectable status & $5(3.9)$ \\
\hline \multicolumn{2}{|l|}{ Tumor burden at ICB administration } \\
\hline Low $(<4 \mathrm{~mL})$ & $35(27.6)$ \\
\hline High ( $\geq 4 \mathrm{~mL}$ ) or unmeasurable/Numerous & $92(72.4)$ \\
\hline \multicolumn{2}{|l|}{ No. of previous systemic therapies before ICB } \\
\hline 0 & $80(63.0)$ \\
\hline 1 & $24(18.9)$ \\
\hline$\geq 2$ & $23(18.1)$ \\
\hline \multicolumn{2}{|l|}{ ICB type } \\
\hline CTLA-4 inhibitor (ipilimumab) & $31(24.4)$ \\
\hline PD-1 inhibitor (pembrolizumab) & $90(70.9)$ \\
\hline PD-1 inhibitor (nivolumab) & $6(4.7)$ \\
\hline Cycles of ICB, median (range) & $3(1-33)$ \\
\hline \multicolumn{2}{|l|}{ Palliative RT administration } \\
\hline No & $71(55.9)$ \\
\hline Yes & $56(44.1)$ \\
\hline
\end{tabular}

ICB, immune checkpoint blocker; CTLA-4, cytotoxic T lymphocyte antigen 4; PD-1, programmed death 1; RT, radiotherapy.

\section{Results}

\section{Patient, tumor, and treatment characteristics}

We identified 127 Korean patients with metastatic melanoma, of whom $103(81.1 \%), 20(15.7 \%)$, and four (3.1\%) were
Table 2. Details of palliative RT in Korean patients with metastatic melanoma who received immune checkpoint blockers with palliative irradiation

\begin{tabular}{|c|c|}
\hline Characteristic & No. $(\%)(n=56)$ \\
\hline $\begin{array}{l}\text { Interval period between ICB and RT, } \\
\text { median (range, mo) }\end{array}$ & $0(0-3)$ \\
\hline Concurrent & $42(75.0)$ \\
\hline Sequential, $\leq 2$ wk & $6(10.7)$ \\
\hline Sequential, $>2 \mathrm{wk}, \leq 3 \mathrm{mo}$ & $8(14.2)$ \\
\hline \multicolumn{2}{|l|}{ RT scheme $^{\text {a) }}$} \\
\hline Conventional & $29(51.8)$ \\
\hline Hypofractionated/SBRT & $27(48.2)$ \\
\hline Total dose, median (range, Gy) & $40(20-75)$ \\
\hline Fractional dose, median (range, Gy) & $4(1.65-15)$ \\
\hline \multicolumn{2}{|l|}{ Dose scheme, total dose/Fractional dose } \\
\hline 33-50 Gy / 1.6-2 Gy & $5(8.9)$ \\
\hline 20-75 Gy/2.1-4.5 Gy & $24(42.9)$ \\
\hline 21-60 Gy/5-9 Gy & $23(41.1)$ \\
\hline 30-45 Gy / 10-15 Gy & $4(7.1)$ \\
\hline \multicolumn{2}{|l|}{ No. of RT sites } \\
\hline Single & $31(55.4)$ \\
\hline Multiple $(\geq 2)$ & $25(44.6)$ \\
\hline \multicolumn{2}{|l|}{ RT site } \\
\hline Brain/Bone only & $13(23.2)$ \\
\hline Parenchymal sites with/Without brain/Bone & $43(76.8)$ \\
\hline \multicolumn{2}{|l|}{ Specific RT site ${ }^{\mathrm{b})}$} \\
\hline Brain & $4(7.1)$ \\
\hline Bone & $21(37.5)$ \\
\hline Skin/Soft tissue & $10(17.9)$ \\
\hline Lung/Chest & $6(10.7)$ \\
\hline Liver/Abdomen & 7 (12.5) \\
\hline Pelvic cavity & $6(10.7)$ \\
\hline Nodal area & $24(42.9)$ \\
\hline Head and neck & $10(17.9)$ \\
\hline Others & $1(1.8)$ \\
\hline
\end{tabular}

$\mathrm{RT}$, radiotherapy; ICB, immune checkpoint blocker; SBRT,

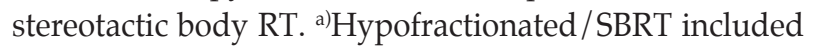
a fractional dose of $5 \mathrm{~Gy}$ or more, ${ }^{\mathrm{b}}$ The number of RT courses during ICB therapy or within 3 months before and after ICB therapy was 89 .

diagnosed with acral/mucosal, uveal, and CSD subtypes. The median age at referral for ICB therapy was 60 years (range, 19 to 89 years). Eight patients (6.3\%) exhibited brain metastasis. ICB monotherapy was administered to 71 patients (55.9\%); the remaining 56 patients $(44.1 \%)$ received combination therapy with ICB and palliative RT. Twenty patients (15.7\%) underwent salvage or palliative resection of the metastatic tumor, of whom 11 showed no evident disease at the time of ICB administration. 


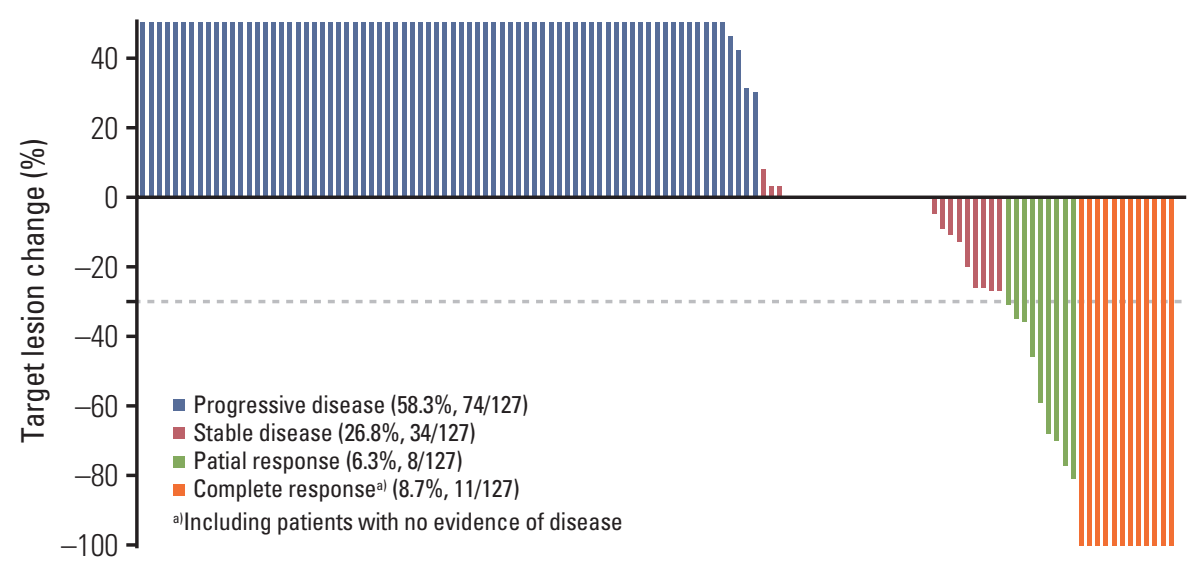

Fig. 1. Waterfall plot for the overall objective response rate in Korean patients with metastatic melanoma who received immune checkpoint blockers.

Table 3. Factors related to the objective response rate in Korean patients with metastatic melanoma who received immune checkpoint blockers

\begin{tabular}{|c|c|c|c|c|}
\hline \multirow{2}{*}{ Characteristic } & \multicolumn{2}{|c|}{ Univariate analysis } & \multicolumn{2}{|c|}{ Multivariate analysis ${ }^{\text {b) }}$} \\
\hline & ORR $^{\text {a) }}(\%)$ & p-value & Odds ratio $(95 \% \mathrm{CI})$ & p-value \\
\hline \multicolumn{5}{|l|}{ Subtype } \\
\hline Chronic sun damage & 50.0 & 0.009 & Ref & 0.167 \\
\hline Uvea & 0.0 & & & 0.998 \\
\hline Acral/Mucosal & 16.5 & & $0.07(0.01-1.10)$ & 0.059 \\
\hline \multicolumn{5}{|l|}{ Stage } \\
\hline M1a/Unresectable & 36.1 & $<0.001$ & Ref & 0.009 \\
\hline M1b/M1c/M1d & 6.6 & & $0.19(0.06-0.66)$ & \\
\hline \multicolumn{5}{|l|}{$B R A F$ status } \\
\hline Wild type & 16.9 & 0.339 & - & - \\
\hline V600 mutation & 12.5 & & - & \\
\hline Unknown & 9.1 & & - & \\
\hline \multicolumn{5}{|l|}{ Tumor burden } \\
\hline Low $(<4 \mathrm{~mL})$ & 37.1 & $<0.001$ & Ref & 0.008 \\
\hline High ( $\geq 4 \mathrm{~mL}$ ) or unmeasurable/Numerous & 6.5 & & $0.20(0.06-0.66)$ & \\
\hline \multicolumn{5}{|l|}{ No. of previous systemic therapies before ICB } \\
\hline 0 & 18.8 & 0.118 & - & - \\
\hline$\geq 1$ & 8.5 & & - & \\
\hline \multicolumn{5}{|l|}{ ICB type } \\
\hline CTLA-4 inhibitor & 3.2 & 0.042 & Ref & 0.075 \\
\hline PD-1 inhibitor & 18.8 & & $16.24(0.75-350.36)$ & \\
\hline \multicolumn{5}{|l|}{ Palliative RT administration } \\
\hline No & 12.7 & 0.416 & - & - \\
\hline Yes & 17.9 & & - & \\
\hline
\end{tabular}

ORR, objective response rate; $\mathrm{CI}$, confidential interval; Ref, reference; ICB, immune checkpoint blocker; CTLA-4, cytotoxic $\mathrm{T}$

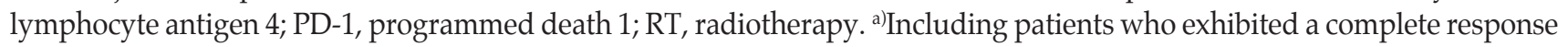
or partial response, ${ }^{\mathrm{b})}$ All variables were analyzed using the binary logistic regression model with a backward stepwise method if $\mathrm{p} \leq 0.10$, and they were removed when $\mathrm{p}>0.10$. 
Among the 127 patients, 31 received ipilimumab for CTLA-4 inhibition and 96 received pembrolizumab $(\mathrm{n}=90)$ or nivolumab ( $\mathrm{n}=6)$ for PD-1 inhibition. One to 33 cycles of ICB therapy were administered, with a median of three cycles. Eighty patients $(64 \%)$ received ICB therapy as first-line systemic therapy after recurrence $(n=64)$ or initial therapy after diagnosis $(n=16)$. The patient, tumor, and treatment characteristics are summarized in Table 1 . Among the patients who received combination therapy, $86 \%$ received RT concurrently or within 2 weeks after ICB administration. A three-dimensional conformal technique $(\mathrm{n}=29)$ or hypofractionated/stereotactic body RT ( $\mathrm{n}=27)$ was used. RT was performed at various sites with a median total dose of $40 \mathrm{~Gy}$ (range, 20 to $75 \mathrm{~Gy}$ ) and a median fractional dose of $4 \mathrm{~Gy}$ (range, 1.65 to $15 \mathrm{~Gy})$. The detailed characteristics of RT are summarized in Table 2.

\section{Response}

The overall ORR after ICB therapy was $15.0 \%$, with 11 CRs and eight PRs. The response rate and overall percentage change from baseline for all patients are shown in Fig. 1. The median time to a response after ICB administration was 8.0 months (range, 2 to 24 months). None of the patients who received ICB with RT showed an abscopal effect.

Table 3 lists the factors that were significantly associated with ORR. ORRs were $50.0 \%, 0 \%$, and $16.5 \%$ for the CSD, uveal, and acral/mucosal subtypes, respectively ( $\mathrm{p}=0.009$ ). There was one case each of CR and PR among the patients with CSD melanomas, while 10 exhibited CR and seven exhibited PR among the patients with acral/mucosal melanomas. S1 Table presents details regarding the overall response according to each subtype. ORR was significantly higher in patients with M1a/unresectable disease than in those with M1b/M1c/M1d disease at the time of ICB administration ( $36.1 \%$ vs. $6.6 \%, \mathrm{p}<0.001)$. Moreover, ORR was significantly higher in patients with a low tumor burden $(<4 \mathrm{~mL})$ than in those with a high tumor burden $(\geq 4 \mathrm{~mL}$ or numerous/ unmeasurable metastatic tumors; $37.1 \%$ vs. $6.5 \%, \mathrm{p}<0.001$ ) at the time of ICB administration. With regard to ORR according to the ICB type, patients who received PD-1 blockers exhibited a significantly higher ORR than those who received CTLA- 4 blockers ( $18.8 \%$ vs. $3.2 \%, p=0.042)$. Palliative RT administration did not have a significant influence on ORR $(\mathrm{p}=0.416)$. In the multivariate analysis, both stage $(\mathrm{p}=0.009)$ and tumor burden $(\mathrm{p}=0.008)$ at ICB administration influenced ORR. Additionally, acral/mucosal subtype had a lower ORR than the CSD subtype, with an odds ratio of 0.07 and borderline significance $(\mathrm{p}=0.059)$ in the multivariate analysis.

We assessed the RT-related factors that influenced ORR in patients who received ICBs with RT ( $\mathrm{n}=56)$. None of the factors exhibited a statistically significant effect. In this cohort, we intended to extract variables associated with the combined treatment approach that enhanced the anti-melanoma effect. Accordingly, we analyzed ORR according to the interval between ICB and RT administration, which ranged from 2 to 12 weeks, to investigate the appropriate interval associated with an improvement in the ICB effectiveness. Among them, the overall ORR tended to improve when RT was administered within 2 weeks of ICB ( $\leq 2$ weeks vs. $>2$ weeks; $20.8 \%$ vs. $0 \%, \mathrm{p}=0.154)$. Furthermore, we found that the ORR increased as the interval between ICB and RT administration decreased (Fig. 2A). Additionally, patients who received RT at parenchymal sites exhibited a higher ORR than those who received brain or bone $\mathrm{RT}$ ( $20.9 \%$ vs. $7.7 \%, \mathrm{p}=0.275)$ (Fig. 2B).

A
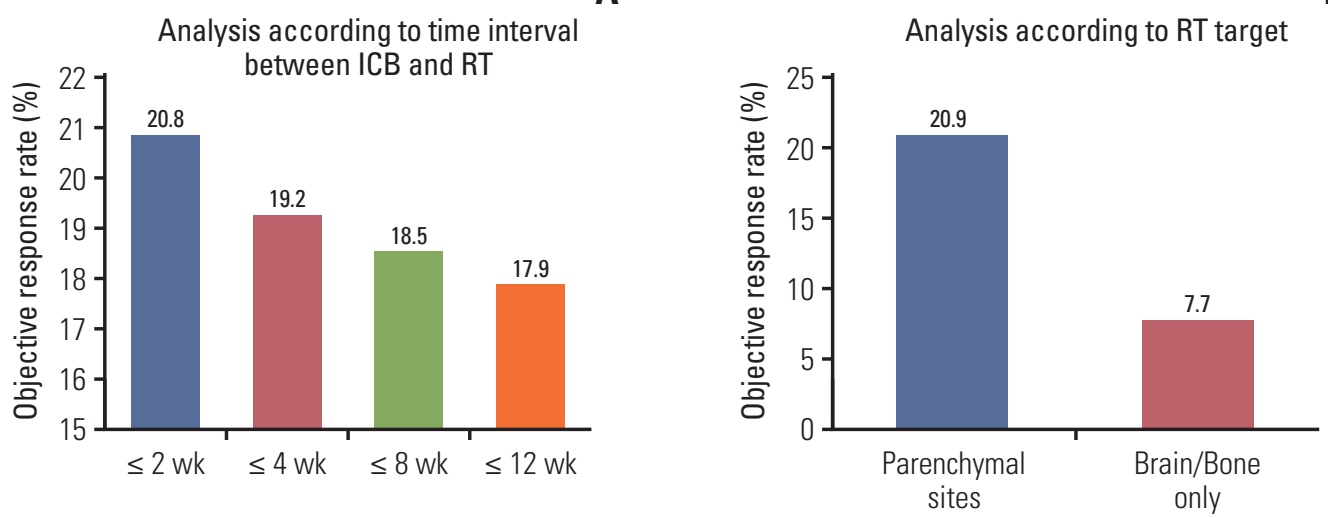

Fig. 2. Radiotherapy (RT)-related factors that influenced the objective response rate in Korean patients with metastatic melanoma who received immune checkpoint blockers (ICBs) with RT ( $n=56)$ according to the interval between ICB and RT administration (A) and the site of RT (B). 


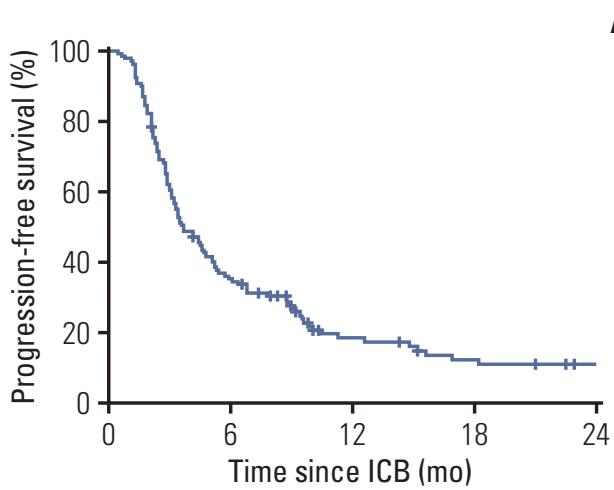

A
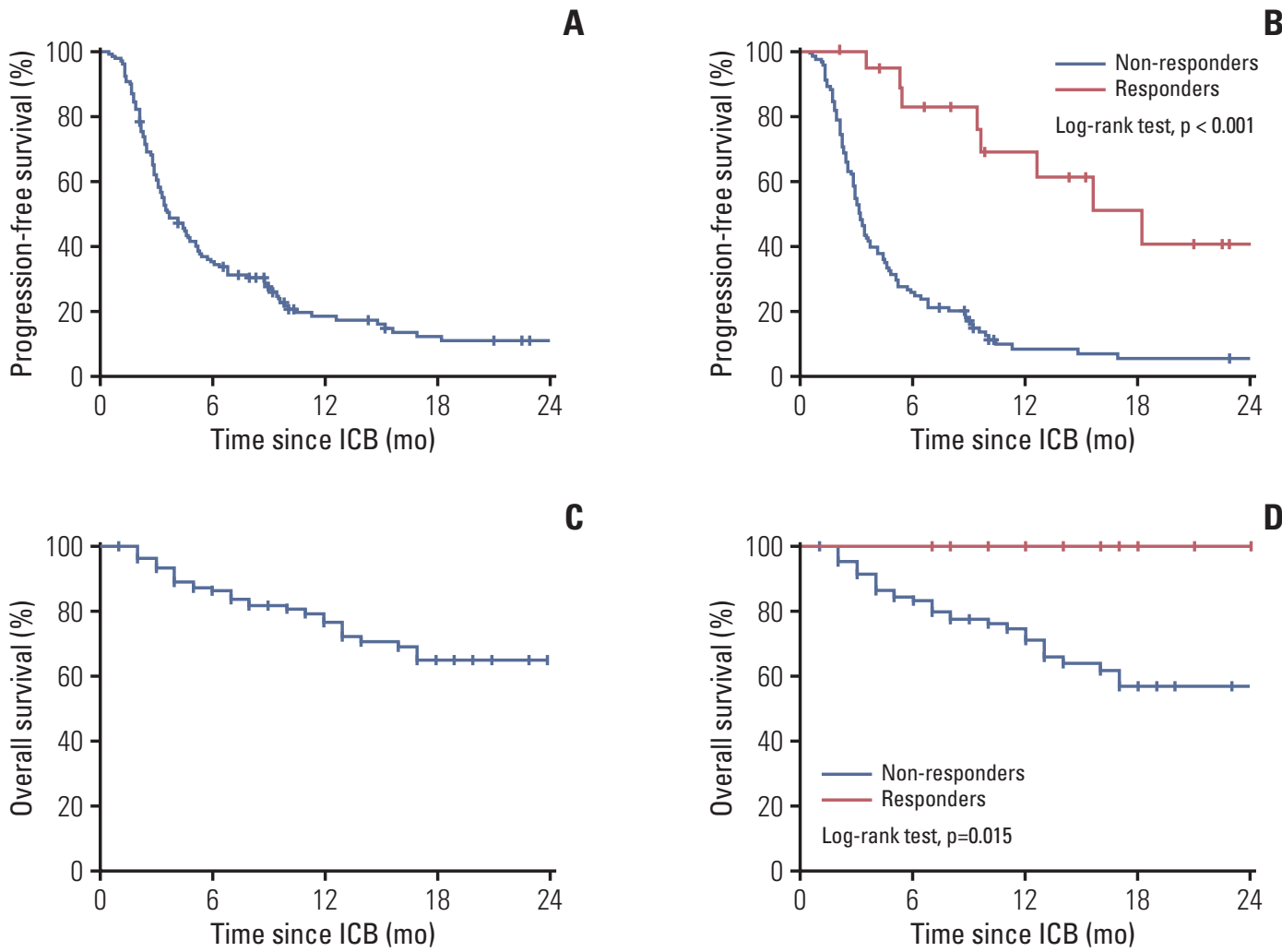

Fig. 3. Kaplan-Meier estimates of survival in Korean patients with metastatic melanoma who received immune checkpoint blockers (ICBs). (A, B) Progression-free survival for all patients (A) and according to the objective response (responders vs. nonresponders) (B). (C, D) Overall survival for all patients (C) and according to the objective response (responders vs. nonresponders) (D).

Table 4. Treatment-related adverse events in Korean patients with metastatic melanoma who received immune checkpoint blockers

\begin{tabular}{|c|c|c|c|c|}
\hline Adverse event & Total $(n=127)$ & ICB $(n=71)$ & ICB-RT $(n=56)$ & p-value \\
\hline Skin rash, G1/2 & $5(3.9)$ & $4(5.6)$ & $1(1.8)$ & 0.468 \\
\hline Pruritus, G1/2 & $1(0.8)$ & $1(1.4)$ & - & - \\
\hline Vitiligo, G1/2 & $2(1.6)$ & $2(2.8)$ & - & - \\
\hline Hypothyroidism, G1 / 2 & $3(2.4)$ & $3(4.2)$ & - & - \\
\hline AST / ALT elevation, G3/4 & $2(1.6)$ & - & $2(3.6)$ & - \\
\hline Any grade & $13(10.2)$ & $10(14.1)$ & $3(5.4)$ & 0.144 \\
\hline G3-4 $4^{\text {a) }}$ & $2(1.6)$ & 0 & $2(3.6)$ & 0.192 \\
\hline
\end{tabular}

Values are presented as number (\%). ICB, immune checkpoint blocker; RT, radiotherapy; AST, aspartate aminotransferase; ALT, alanine aminotransferase; G, toxicity grade. ${ }^{\text {a) }}$ There was no grade 5 adverse event.

\section{Survival}

The median follow-up duration for the entire cohort was 11 months (range, 1 to 57 months). In total, 108 patients $(85.0 \%)$ experienced disease progression, with a 1-year PFS rate of $17.2 \%$ (median period, 3.6 months; range, 2.7 to 4.5 months) (Fig. 3A). Patients who responded to ICB therapy exhibited a 1-year PFS rate of $61.2 \%$ (median period, 18.2 months; range, 10.4 to 26.0 months), whereas nonresponders exhibited a 1-year PFS rate of $8.7 \%$ (median period, 3.1 
months; range, 2.7 to 3.5 months) (Fig. 3B). The difference between responders and nonresponders was statistically significant $(\mathrm{p}<0.001)$. Thirty-four of the 127 patients $(26.4 \%)$ died during follow-up. The 1-year OS rate was $76.1 \%$, and the median OS was not reached (Fig. 3C). The 1-year OS rate was significantly higher in responders $(100 \%)$ than in nonresponders (71.2\%, $\mathrm{p}=0.015$ ) (Fig. 3D).

\section{Treatment-related adverse events}

Acute adverse events were reported in 13 of the 127 patients (10.2\%), with two patients (1.6\%) developing grade 3 or grade 4 hepatic toxicity. Specifically, 10 patients with acute adverse events $(14.1 \%)$ were identified in the ICB monotherapy group, although none of them exhibited grade 3 or grade 4 toxicity. In the combination therapy group, three patients $(5.4 \%)$ had acute adverse events and two $(3.6 \%)$ exhibited grade 3 or grade 4 hepatic toxicity characterized by elevated aspartate aminotransferase and alanine aminotransferase levels. There was no correlation between the dose and / or fractionation and adverse event development. Accordingly, there was no significant intergroup difference in the incidence of adverse events of any grade $(\mathrm{p}=0.144)$ or grade 3 or grade 4 adverse events $(\mathrm{p}=0.192)$. Table 4 presents the details of all reported adverse events.

\section{Discussion}

We described the outcomes of ICB therapy with or without RT in 127 Korean patients with metastatic melanoma at a single institution. The acral / mucosal, uveal, and CSD subtypes accounted for $81 \%, 16 \%$, and $3 \%$ cases, respectively. The overall ORR, calculated on the basis of the iRECIST criteria, was $15 \%$, while ORRs for the acral/mucosal, uveal, and CSD subtypes were $16.5 \%, 0 \%$, and $50 \%$, respectively. Moreover, clinical responders to ICB therapy exhibited significantly better survival outcomes than nonresponders. Additionally, RT performed concurrently with ICB or within a short interval of time ( $\leq 2$ weeks) tended to enhance the anti-melanoma activity of ICB.

According to a previous study, there are distinct genetic pathways in the development of the different melanoma subtypes [4]. In the present study, the low ORR after ICB therapy could be attributed to the modest response to the acral/ mucosal subtype. In particular, acral melanoma is a skin malignancy with a low mutational burden that negatively influences the response to ICB therapy and results in poor prognosis relative to that of the other subtypes [4-6,16,17]. Furthermore, the incidence of the different subtypes of melanoma differs according to ethnicity. While Caucasians primarily develop non-acral melanoma associated with chronic sun exposure [3,17], Asians and Africans primarily develop acral or mucosal melanomas that predominantly occur in sun-protected areas $[5,18]$. Therefore, acral melanoma represents the smallest subgroup of melanoma worldwide. Previous studies predominantly evaluated the effectiveness of ICB in CSD melanomas and reported an ORR of $30 \%-40 \%$ [7]. On the other hand, our study reported an ORR of $17 \%$, and a study from Japan reported an ORR of $20 \%$ after ICB administration for acral or mucosal melanoma [19]. To date, the precise efficacy of ICB therapy for this melanoma subtype has not been elucidated because of its rarity. Further trials with a large number of Asian patients diagnosed with acral or mucosal melanoma are necessary to establish definitive conclusions.

Over the past two decades, the therapeutic paradigm for metastatic melanoma has rapidly changed with ICB therapy $[1,2]$. Responsiveness to ICB is considered to improve the PFS and OS rates, as observed in the present study where responders exhibited higher PFS and OS rates than nonresponders. In fact, the responders and nonresponders exhibited marked differences of 53\% and 30\% in the 1-year PFS and OS rates, respectively. However, the efficacy of ICB monotherapy remains moderate [7]. The lack of tumor-associated antigens, insufficient infiltration of cytotoxic $\mathrm{T}$ lymphocytes, bulky tumors, and other immunosuppressive pathways are the main causes of resistance to ICB therapy [8]. Several combination treatments for use with ICB, including RT, are being explored in the hope of achieving an enhanced response. RT not only reduces the tumor volume but also exhibits an immunomodulatory effect. It generates a systemic immune effect by increasing tumor antigen uptake and presentation by dendritic cells and subsequently inducing the release of various cytokines, chemokines, and cytotoxic $\mathrm{T}$ cells, thus creating a pro-inflammatory environment $[20,21]$. The recognition of these immunomodulatory effects has led to the integration of RT with ICB for the achievement of enhanced or synergistic anticancer activity [22]. Indeed, clinical studies showed that combination therapy with ICB and RT results in improved antitumor activity [23-25]. A prospective trial showed that $50 \%$ of patients with stage IV melanoma who received palliative RT and ICB exhibited positive clinical outcomes such as CR, PR, and stable disease, with well-tolerated toxicities [23]. In addition, a retrospective study of advanced melanoma observed a CR rate of $25.7 \%$ and a 1-year OS rate of $72 \%$ in patients who received ICB with RT; these values were $6.5 \%$ and $35 \%$, respectively, for patients who received ICB monotherapy [24]. Another recent multicenter study retrospectively evaluated the efficacy of PD-1 inhibitor therapy combined with RT for advanced melanoma and summarized that the OS rate was comparable between patients who recei- 
ved a PD-1 inhibitor with RT and those who received a PD-1 inhibitor alone, despite worse baseline variables for patients who received combination therapy [25].

There is no consensus regarding the appropriate treatment sequence for ICB and RT or selection of the optimal site for enhancing the effects of RT combined with immune therapy. In the present study, we found that ORR tended to improve if RT was performed concurrently with ICB administration or within 14 days of ICB administration, although the results were not statistically significant. In the PACIFIC trial, patients with stage III lung cancer who received durvalumab within 14 days of chemoradiotherapy exhibited a lower hazard ratio for mortality than those who received durvalumab at a later stage [26]. Accordingly, it seems that the close temporal relation between both treatments might contribute to an increase in the anti-melanoma effect. Moreover, we observed that RT at parenchymal sites resulted in a higher ORR than RT at the brain or bone. Additionally, a previous study found that RT at parenchymal sites induced systemic immune changes, whereas RT at nonparenchymal sites did not induce such changes [27]. However, because these results were derived from heterogeneous and uncontrolled cohorts, they should be interpreted with caution.

The incidence of grade 3 or grade 4 adverse events in the present study was only $1.6 \%$. Moreover, compared with ICB monotherapy, combination therapy with ICB and RT did not induce supra-additive toxicity $[25,28]$. In line with our findings, the results of a multicenter study revealed that concurrent PD-1 blocker therapy and RT did not increase or add to previously reported toxicity for PD-1 blockers or RT alone [29]. Notably, because the response to PD-1 blockers is better than that to CTLA-4 blockers, PD- 1 blockers are a better choice than CTLA-4 blockers for combination therapy with RT.

This study had some limitations associated with its retrospective design, including the presence of uncontrolled confounding factors, variations in ICB therapy cycles, variable radiation doses/sites, and under-reporting of toxicity. Moreover, the sample size was relatively small. However, we enrolled a homogenous cohort of patients with metastatic melanoma who received ICB therapy with or without RT at a single institution. Furthermore, we reported the actual effectiveness of ICB for acral/mucosal melanomas in Korean patients and demonstrated that patients with advanced or M1a disease, those with a low tumor burden, and those who received PD-1 blockers exhibited a significant response to ICB with or without RT. Although we could not demonstrate the superiority of combination therapy over ICB monotherapy, we believe that combination therapy can show promising antitumor effects if the optimal treatment sequence, RT site, and RT dose / fractionation are defined in future studies on the basis of past findings [23-25].

In conclusion, we found that acral and mucosal melanomas, which are predominantly observed in Asian populations, exhibited a relatively modest objective response to ICB monotherapy. Such unsatisfactory results have encouraged physicians to combine ICB therapy with other treatments that can enhance its effectiveness. Although we only observed a trend of improvement in ORR after administration of ICB with palliative RT performed within a short interval or RT targeting parenchymal sites, our findings provided a rationale for the initiation of our phase II study to investigate whether immunotherapy plus RT exhibits increased antitumor activity in patients with melanoma (NCT04017897) [30].

\section{Electronic Supplementary Material}

Supplementary materials are available at Cancer Research and Treatment website (https:// www.e-crt.org).

\section{Conflicts of Interest}

Conflict of interest relevant to this article was not reported.

\section{Acknowledgments}

This study was supported by a faculty research grant of Yonsei University College of Medicine (6-2018-0163).

\section{References}

1. Postow MA, Chesney J, Pavlick AC, Robert C, Grossmann K, McDermott D, et al. Nivolumab and ipilimumab versus ipilimumab in untreated melanoma. N Engl J Med. 2015;372:200617.

2. Hodi FS, Chesney J, Pavlick AC, Robert C, Grossmann KF, McDermott DF, et al. Combined nivolumab and ipilimumab versus ipilimumab alone in patients with advanced mela- noma: 2-year overall survival outcomes in a multicentre, randomised, controlled, phase 2 trial. Lancet Oncol. 2016;17:155868.

3. Bradford PT, Goldstein AM, McMaster ML, Tucker MA. Acral lentiginous melanoma: incidence and survival patterns in the United States, 1986-2005. Arch Dermatol. 2009;145:427-34.

4. Curtin JA, Fridlyand J, Kageshita T, Patel HN, Busam KJ, 
Kutzner $\mathrm{H}$, et al. Distinct sets of genetic alterations in melanoma. N Engl J Med. 2005;353:2135-47.

5. Shoushtari AN, Munhoz RR, Kuk D, Ott PA, Johnson DB, Tsai KK, et al. The efficacy of anti-PD-1 agents in acral and mucosal melanoma. Cancer. 2016;122:3354-62.

6. Hayward NK, Wilmott JS, Waddell N, Johansson PA, Field MA, Nones K, et al. Whole-genome landscapes of major melanoma subtypes. Nature. 2017;545:175-80.

7. Ribas A, Wolchok JD. Cancer immunotherapy using checkpoint blockade. Science. 2018;359:1350-5.

8. Wei Y, Du Q, Jiang X, Li L, Li T, Li M, et al. Efficacy and safety of combination immunotherapy for malignant solid tumors: a systematic review and meta-analysis. Crit Rev Oncol Hematol. 2019;138:178-89.

9. Pfirschke C, Engblom C, Rickelt S, Cortez-Retamozo V, Garris C, Pucci F, et al. Immunogenic chemotherapy sensitizes tumors to checkpoint blockade therapy. Immunity. 2016;44: 343-54.

10. Fukumura D, Kloepper J, Amoozgar Z, Duda DG, Jain RK. Enhancing cancer immunotherapy using antiangiogenics: opportunities and challenges. Nat Rev Clin Oncol. 2018;15: 325-40.

11. Demaria S, Golden EB, Formenti SC. Role of local radiation therapy in cancer immunotherapy. JAMA Oncol. 2015;1:132532.

12. Vanpouille-Box C, Alard A, Aryankalayil MJ, Sarfraz Y, Diamond JM, Schneider RJ, et al. DNA exonuclease Trex1 regulates radiotherapy-induced tumour immunogenicity. Nat Commun. 2017;8:15618.

13. Shargel L, Wu-Pong S, Yu AB. Applied biopharmaceutics and pharmacokinetics. 7th ed. New York: McGraw-Hill Education; 2015.

14. Seymour L, Bogaerts J, Perrone A, Ford R, Schwartz LH, Mandrekar S, et al. iRECIST: guidelines for response criteria for use in trials testing immunotherapeutics. Lancet Oncol. 2017;18: e143-52.

15. Somarouthu B, Lee SI, Urban T, Sadow CA, Harris GJ, Kambadakone A. Immune-related tumour response assessment criteria: a comprehensive review. Br J Radiol. 2018;91:20170457.

16. Tas F, Erturk K. Acral lentiginous melanoma is associated with certain poor prognostic histopathological factors but may not be correlated with nodal involvement, recurrence, and a worse survival. Pathobiology. 2018;85:227-31.

17. Bello DM, Chou JF, Panageas KS, Brady MS, Coit DG, Carvajal RD, et al. Prognosis of acral melanoma: a series of 281 patients. Ann Surg Oncol. 2013;20:3618-25.

18. Roh MR, Kim J, Chung KY. Treatment and outcomes of melanoma in acral location in Korean patients. Yonsei Med J. 2010;51:562-8.

19. Maeda T, Yoshino K, Nagai K, Oaku S, Kato M, Hiura A, et al. Efficacy of nivolumab monotherapy against acral lentiginous melanoma and mucosal melanoma in Asian patients. Br J Dermatol. 2019;180:1230-1

20. Reits EA, Hodge JW, Herberts CA, Groothuis TA, Chakraborty M, Wansley EK, et al. Radiation modulates the peptide repertoire, enhances MHC class I expression, and induces successful antitumor immunotherapy. J Exp Med. 2006;203:125971.

21. Lhuillier C, Rudqvist NP, Elemento O, Formenti SC, Demaria S. Radiation therapy and anti-tumor immunity: exposing immunogenic mutations to the immune system. Genome Med. 2019;11:40.

22. Twyman-Saint Victor C, Rech AJ, Maity A, Rengan R, Pauken KE, Stelekati E, et al. Radiation and dual checkpoint blockade activate non-redundant immune mechanisms in cancer. Nature. 2015;520:373-7.

23. Hiniker SM, Reddy SA, Maecker HT, Subrahmanyam PB, Rosenberg-Hasson Y, Swetter SM, et al. A prospective clinical trial combining radiation therapy with systemic immunotherapy in metastatic melanoma. Int J Radiat Oncol Biol Phys. 2016;96:578-88.

24. Koller KM, Mackley HB, Liu J, Wagner H, Talamo G, Schell $\mathrm{TD}$, et al. Improved survival and complete response rates in patients with advanced melanoma treated with concurrent ipilimumab and radiotherapy versus ipilimumab alone. Cancer Biol Ther. 2017;18:36-42.

25. Mowery YM, Patel K, Chowdhary M, Rushing CN, Roy Choudhury K, Lowe JR, et al. Retrospective analysis of safety and efficacy of anti-PD-1 therapy and radiation therapy in advanced melanoma: a bi-institutional study. Radiother Oncol. 2019;138:114-20.

26. Antonia SJ, Villegas A, Daniel D, Vicente D, Murakami S, Hui $\mathrm{R}$, et al. Overall survival with durvalumab after chemoradiotherapy in stage III NSCLC. N Engl J Med. 2018;379:2342-50.

27. McGee HM, Daly ME, Azghadi S, Stewart SL, Oesterich L, Schlom J, et al. Stereotactic ablative radiation therapy induces systemic differences in peripheral blood immunophenotype dependent on irradiated site. Int J Radiat Oncol Biol Phys. 2018;101:1259-70.

28. Couty E, Vallard A, Sotton S, Ouni S, Garcia MA, Espenel S, et al. Safety assessment of anticancer drugs in association with radiotherapy in metastatic malignant melanoma: a real-life report: radiation/systemic drug combo in metastatic melanoma. Cancer Chemother Pharmacol. 2019;83:881-92.

29. Amin NP, Zainib M, Parker SM, Agarwal M, Mattes MD. Multi-institutional report on toxicities of concurrent nivolumab and radiation therapy. Adv Radiat Oncol. 2018;3:399404.

30. ClinicalTrials.gov. The combination of anti-PD-1 with radiotherapy in previously untreated metastatic melanoma [Internet]. Bethesda, MD: U.S. National Library of Medicine; 2019 [cited 2019 Aug 19]. Available from: https:// clinicaltrials.gov/ ct2 / show / NCT04017897?cond=NCT04017897\&rank=1. 